\title{
Review \\ Year in review 2008: Critical Care - cardiology
}

Luigi Camporota, Marius Terblanche and David Bennett

Adult Intensive Care Unit, Guy's and St Thomas' NHS Foundation Trust, St Thomas' Hospital, 1st Floor East Wing, Lambeth Palace Road, London, SE1 7EH, UK

Corresponding author: Marius Terblanche, marius.terblanche@gstt.nhs.uk

Published: 21 October 2009

This article is online at http://ccforum.com/content/13/5/229

(c) 2009 BioMed Central Ltd

Critical Care 2009, 13:229 (doi:10.1186/cc8025)

\begin{abstract}
We review key research papers in cardiology and intensive care published during 2008 in Critical Care. We quote studies on the same subject published in other journals if appropriate. Papers have been grouped into three categories: (a) cardiovascular biomarkers in critical illness, (b) haemodynamic management of septic shock, and (c) haemodynamic monitoring.
\end{abstract}

\section{Cardiovascular biomarkers in critical illness Cardiac troponins}

Cardiac troponins (cTns) are highly sensitive and specific biological markers of myocardial damage. Elevated cTn is an independent predictor of adverse outcome and correlates with intensive care unit (ICU) and hospital lengths of stay among critically ill patients, regardless of the mechanism causing its rise [1-3]. However, because ICU patients often have increased cTn for reasons other than overt myocardial infarction (MI), raised $\mathrm{cTn}$ may be attributed to other conditions, and therefore the true incidence of myocardial damage in ICU may be underestimated.

Lim and colleagues [4] screened patients admitted to ICU by using $\mathrm{cTn}$ and electrocardiograms (ECGs) to determine the incidence of elevated cTn and $\mathrm{Ml}$ and to assess whether these findings influence prognosis. In this study, patients were classified as having $\mathrm{Ml}$ in the presence of elevated $\mathrm{cTn}$ and ECG evidence supporting a diagnosis of Ml. Among 103 patients, $35.9 \%$ had a confirmed $\mathrm{Ml}$ whereas $14.6 \%$ had an elevated cTn only. Patients with an Ml or with elevated cTn without ECG changes had a longer duration of mechanical ventilation and ICU stay and higher ICU and hospital mortality rates compared with patients with no cTn elevation (odds ratio 27.3). Lim and colleagues [4] found that screening cTn measurements and 12-lead ECGs detected $\mathrm{Ml}$ at a higher rate than clinical diagnosis alone, suggesting that the true incidence and associated mortality of MI in ICU patients are underestimated.

\section{Brain natriuretic peptides}

Increased levels of brain natriuretic peptide (BNP) and the biologically inactive N-terminal pro-BNP (NT-proBNP) are associated with impaired left ventricular (LV) function and ischaemia, pulmonary embolism (PE) and chronic obstructive pulmonary disease [5].

Coutance and colleagues [6] conducted a meta-analysis of studies in patients with acute PE to assess the prognostic value of elevated BNP or NT-proBNP levels to predict shortterm overall mortality, PE-specific mortality and the occurrence of serious pre-defined adverse events. The study showed that elevated BNP or NT-proBNP levels may help to identify patients with acute PE and right ventricular (RV) dysfunction at high risk of short-term death and adverse outcome events. BNP and NT-proBNP had low positive predictive values (PPVs) for death (14\%) but a high negative predictive value (99\%), suggesting that BNP or NT-proBNP might be useful in identifying patients with a likely favourable outcome.

Kirchhoff and colleagues [7] prospectively studied the relationship between NT-proBNP, disease severity and cardiac output $(\mathrm{CO})$ monitoring measured by transpulmonary

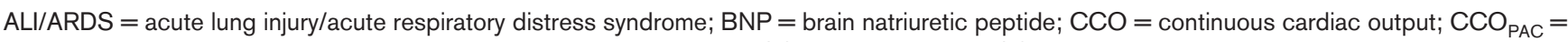
continuous cardiac output by pulmonary artery catheter thermodilution; $\mathrm{CO}=$ cardiac output; $\mathrm{CO}_{\mathrm{TCP}}=$ transcardiopulmonary thermodilution cardiac output; cTn = cardiac troponin; CVP = central venous pressure; ECG = electrocardiogram; IAH = intra-abdominal hypertension; ICU = intensive care unit; LV = left ventricular; LVD = left ventricular systolic or diastolic dysfunction; MI = myocardial infarction; MODS = multiple organ dysfunction syndrome; NT-proBNP $=\mathrm{N}$-terminal pro-brain natriuretic peptide; PAOP $=$ pulmonary artery occlusion pressure; $\mathrm{PCCO}=$ pulse contourderived cardiac output; $\mathrm{PCCO}_{\text {pre }}=$ pre-calibration pulse contour-derived cardiac output; $\mathrm{PE}=$ pulmonary embolism; $\mathrm{pHi}=$ intramucosal $\mathrm{pH}$; $\mathrm{PiCCO}=$ pulse contour cardiac output; $\mathrm{PPV}=$ positive predictive value; $\mathrm{RAP}=$ right atrial pressure; $\mathrm{ROC}=$ receiver operating characteristic; $\mathrm{RV}=$ right ventricular; $\mathrm{ScvO}_{2}=$ central venous oxygen saturation; $\mathrm{SV}=$ stroke volume; $\mathrm{SvO}_{2}=$ mixed venous oxygen saturation; $\mathrm{SVV}=$ stroke volume variation; $\mathrm{SVV}_{\mathrm{FloTrac}}=$ stroke volume variation calculated using the FloTrac/Vigileo ${ }^{\mathrm{TM}}$ system algorithm; $\mathrm{SVV}_{\mathrm{PiCcO}}=$ stroke volume variation calculated using the PiCCO ${ }_{P / u s}{ }^{\text {TM }}$ system; TEE = transoesophageal echocardiography. 
thermodylution (pulse contour cardiac output, or $\mathrm{PiCCO}$ ) in 26 trauma patients with no previous history of cardiac, renal or hepatic impairment. Patients were subdivided into two groups based on disease severity by using the multiple organ dysfunction syndrome (MODS) score: group A had minor organ dysfunction (MODS score $\leq 4$ ) and group $B$ had major organ dysfunction (MODS score $>4$ ). Serum NT-proBNP levels were elevated in all patients. NT-proBNP was significantly lower at baseline and at all subsequent time points in group $A$, whereas the cardiac index was significantly higher in group $A$ at baseline and at all time points. The investigators also found a significant inverse correlation between cardiac index and MODS score and a positive correlation between MODS score and serum NT-proBNP levels. These pilot data hint at a potential value of NT-proBNP in the diagnosis of post-traumatic cardiac impairment.

BNP and NT-proBNP are frequently elevated in critically ill patients and both show a dispersion that is much larger than that of a non-ICU population. Coquet and colleagues [8] conducted a prospective observational study of medical ICU patients to evaluate the accuracy of NT-proBNP as a marker of cardiac dysfunction in a heterogeneous group of critically ill patients. Of 198 patients included, 51.5\% had echocardiographic evidence of cardiac dysfunction. Median NT-proBNP concentrations were 6.7 times higher in patients with cardiac dysfunction (area under the receiver operating characteristic [ROC] curve 0.76). While adding ECG changes and organ failure score increased the area under the ROC curve to 0.83 , NT-ProBNP was not independently associated with outcome. Despite the effects of age and creatinine clearance on NTproBNP levels, a single measurement of the NT-proBNP level at ICU admission might rule out cardiac dysfunction in critically ill patients independently of age or renal function.

BNP or NT-proBNP may theoretically be useful in distinguishing pulmonary oedema due to acute lung injury/acute respiratory distress syndrome (ALI/ARDS) from hydrostatic or cardiogenic oedema. Levitt and colleagues [9] performed a prospective blinded cohort study in a mixed medical and surgical ICU to assess the diagnostic utility of BNP in a cohort of ventilated patients with convincing evidence of either ALI/ARDS or cardiogenic pulmonary oedema. BNP was measured immediately after enrolment and then daily for 3 days in patients with ALI/ARDS (defined as a pulmonary artery occlusion pressure [PAOP] of less than $16 \mathrm{~mm} \mathrm{Hg}$ or a right atrial pressure [RAP] of less than $10 \mathrm{~mm} \mathrm{Hg}$ and no echocardiographic evidence of new or worsening left ventricular systolic or diastolic dysfunction [LVD]) and in patients with cardiogenic oedema (defined as a PAOP of greater than $20 \mathrm{~mm} \mathrm{Hg}$ or an RAP of greater than $14 \mathrm{~mm} \mathrm{Hg}$ with a current echocardiogram documenting new or worsening LVD) [9].

BNP levels (at baseline and with repeated measurement) did not reliably distinguish ALI/ARDS from cardiogenic pulmonary oedema despite the efforts to clearly separate the two groups based on haemodynamic parameters. Given that ALI/ARDS and cardiac dysfunction are not mutually exclusive conditions, the clinical utility of BNP testing in this setting may well be limited [9].

\section{Lactates and central venous oximetry}

Lactates and central venous oxygen saturation $\left(\mathrm{ScvO}_{2}\right)-$ measured from the superior vena cava - are used as indicators of adequacy of tissue oxygen supply. Patients with high lactates, even in the absence of hypotension ('occult shock'), are at higher mortality risk. In patients with severe sepsis/shock and raised lactate levels, directing treatment to target $\mathrm{ScvO}_{2}$ is associated with a significant survival benefit [10]. However, the key factor for improving survival is early recognition and intervention.

In a prospective observational pilot study of 124 patients requiring urgent pre-hospital care, Jansen and colleagues [11] studied the relationship between pre-hospital capillary or venous lactate levels and in-hospital mortality. Lactate levels were measured by the Emergency Medical Services (using a handheld device) on arrival at the scene (T1) and just before or on arrival at the emergency department (T2). Mortality was higher in those with $\mathrm{T} 1$ lactate of greater than $3.5 \mathrm{mmol} / \mathrm{L}$ compared with a lactate of less than $3.5 \mathrm{mmol} / \mathrm{L}$ (T1: $41 \%$ versus 12\%; T2: 47\% versus 15\%). In multivariate analysis, only delta lactate and GCS were significantly associated with mortality, with hazard ratios (95\% confidence intervals) of 0.2 (0.05 to 0.76$)$ and 0.93 (0.88 to 0.99$)$, respectively. These pilot data suggest that it may be possible to identify a group of patients at high risk before admission to hospital and that appropriate management at this very early stage may improve outcomes.

Low $\mathrm{ScvO}_{2}$ values have also been associated with an increased risk of postoperative complications in high-risk surgery [12] and in severe sepsis [10]. Little is known, however, of the $\mathrm{ScvO}_{2}$ profile of other patient groups. In an unselected group of unplanned ICU admissions, Bracht and colleagues [13] showed that, on ICU admission, $21 \%$ of patients had an $\mathrm{ScvO}_{2}$ of less than $60 \%$. In this group, an $\mathrm{ScvO}_{2}$ of less than $60 \%$ was associated with an increased mortality $(29 \%$ versus $17 \%, P<0.05)$ but not with ICU or hospital length of stay [13]. The mean \pm standard deviation value of $\mathrm{ScvO}_{2}$ for the septic group was $68 \% \pm 12 \%$, significantly higher than the $49 \%$ reported by Rivers and colleagues [10].

To compare ICU admission $\mathrm{ScvO}_{2}$ values in Dutch ICUs with data from Rivers and colleagues [10], van Beest and colleagues [14] performed a prospective observational multicentre study. While the 'incidence' of low $\mathrm{ScvO}_{2}$ is reported, the data actually reflect its 'prevalence'. The mean mixed venous oxygen saturation $\left(\mathrm{SvO}_{2}\right)$ and $\mathrm{ScvO}_{2}$ values were greater than $65 \%$ and greater than $70 \%$, respectively. Only 
$14 \%$ and $5 \%$ of the overall population had $\mathrm{ScvO}_{2}$ values of less than $60 \%$ and less than 50\%, respectively. Among septic patients, the prevalence of an $\mathrm{ScvO}_{2}$ of less than $60 \%$ and less than $50 \%$ was even lower $(6 \%$ and $1 \%$, respectively), highlighting that the syndrome described by Rivers and colleagues [10] may be relatively uncommon in the ICU depending on the specific hospital setting but remains important as patients with an $\mathrm{ScvO}_{2}$ of less than $50 \%$ exhibited the highest in-hospital mortality (57\%) compared with an overall mortality of 32\% [14]. These data raise concerns about the utility of an $\mathrm{ScvO}_{2}$-guided therapy in severe sepsis patients admitted to ICU, as opposed to the emergency department, and will require further studies [15], particularly as $\mathrm{SvO}_{2}$ and $\mathrm{ScvO}_{2}$ are only indirect indices of global tissue oxygenation and do not provide any insight on the state of oxygen utilization in tissues [16].

In summary:

1. When used as a screening tool in critically ill patients, cTn and 12-lead ECG lead to a higher rate of MI diagnosis.

2. BNP or NT-proBNP levels may help to identify patients with acute $\mathrm{PE}$ and RV dysfunction with a likely favourable outcome, while a single measurement of the NT-proBNP level at ICU admission might rule out cardiac dysfunction in critically ill patients independently of age or renal function.

3. However, BNP or NT-proBNP levels do not distinguish ALI/ARDS from cardiogenic pulmonary oedema.

4. Both high lactates and low $\mathrm{ScvO}_{2}$ are associated with higher mortality, but the percentage of ICU patients with an $\mathrm{ScvO}_{2}$ of less than $50 \%$ is small.

\section{Haemodynamic management of septic shock}

Low gastric intramucosal $\mathrm{pH}(\mathrm{pHi})$ is a sensitive marker of splanchnic hypoperfusion and a good predictor of poor outcome in critically ill patients. In a randomised trial, Palizas and colleagues [17] studied 30 septic shock patients who were randomly assigned within 48 hours of ICU admission to two different resuscitation goals: $\mathrm{Cl}$ of greater than 3.0 L/minute per square metre or $\mathrm{pHi}$ of greater than 7.32 [17]. Although there was no difference in the primary endpoint (28-day mortality and ICU length of stay), a higher proportion of patients exhibited values below the specific target at baseline in the pHi group compared with the $\mathrm{Cl}$ group (50\% versus $10.9 \%$ ). Of 32 patients with a pHi of less than 7.32 at baseline, only $22 \%$ had a pHi of greater than 7.32 after resuscitation. Areas under the ROC curves to predict mortality at baseline and at 24 and 48 hours were 0.70 versus 0.55 , 0.9 versus 0.61 , and 0.75 versus 0.47 , respectively, demonstrating that a normalization of $\mathrm{pHi}$ within 24 hours of resuscitation is a strong signal of therapeutic success, and in contrast, a persistent low pHi despite treatment is associated with a very poor prognosis in septic shock patients [17]. The study, powered to detect a $20 \%$ absolute risk reduction in 28 day mortality, likely suffers from residual confounding or bias or both. For example, while there was no difference in the primary endpoint, only $22 \%$ of those in the pHi group had a $\mathrm{pHi}$ of greater than 7.32 after resuscitation.

Gastrointestinal mucosal perfusion can be affected by the type of vasopressor used for the initial management of septic shock. In a prospective randomized controlled trial of patients with septic shock, Morelli and colleagues [18] showed that first-line therapy with phenylephrine does not worsen hepatosplanchnic perfusion during initial haemodynamic support of patients with septic shock and that phenylephrine had effects similar to those of norepinephrine on cardiopulmonary performance and global oxygen transport and similar effects on creatinine clearance, although higher doses were required to achieve the same target of mean arterial pressure of $70 \pm 5 \mathrm{~mm} \mathrm{Hg}$ [18].

The rate of weaning of vasopressor drugs in patients with septic shock is usually an empirical choice made by the clinician. However, in a prospective randomised trial, Merouani and colleagues [19] applied a closed-loop control based on fuzzy logic principles to titrate intravenous norepinephrine (noradrenaline) infusion rates in septic patients in order to reduce the duration of poorly controlled haemodynamic status. Septic patients were randomly assigned to norepinephrine infused either at the clinician's discretion (control group) or under closed-loop control based on fuzzy logic (fuzzy group). The infusion rate changed automatically after analysis of mean arterial pressure in the fuzzy group. The fuzzy group had a shorter median duration of vasoactive drugs (28.5 versus 57.5 hours) and a lower total amount of norepinephrine infused during shock ( 0.6 versus $1.4 \mu \mathrm{g} / \mathrm{kg}$ ); however, no difference in terms of mortality or duration of mechanical ventilation was found.

During septic shock, resistance to the haemodynamic effects of catecholamine vasopressors and inotropes is a marker of mortality risk, and the metabolic and haemodynamic responses to dobutamine may correlate with outcome in patients with septic shock. In a prospective, non-randomised, non-blinded interventional study of patients with severe sepsis or septic shock who underwent a graded dobutamine challenge (5 to $20 \mu \mathrm{g} / \mathrm{kg}$ per minute), Kumar and colleagues [20] showed that survival from severe sepsis or septic shock is associated with increased cardiac performance and contractility indices during dobutamine infusion.

In multivariate analysis, an increase in stroke volume (SV) index of greater than $8.5 \mathrm{~mL} / \mathrm{m}^{2}$ was the dominant discriminating variable for survival. The fact that both cardiac performance and contractility increases in response to dobutamine are highly associated with outcome in septic shock suggests that the inability to recruit ventricular volume during cardiovascular stress may indicate that the heart is already operating at the maximum efficacy of the FrankStarling response and therefore has no additional reserve. 
Catecholamines and inflammatory mediators play a significant role in the pathogenesis of septic cardiomyopathy, and there is growing evidence of an association between beta-adrenergic stress and the pathogenesis of septic cardiomyopathy. Beta-blockers in critically ill patients may thus attenuate cathecholamine-induced myocardial stunning and septic cardiomyopathy. In a retrospective analysis of the combined use of milrinone and enteral metoprolol therapy in 40 patients with septic shock and cardiac depression, Schmittinger and colleagues [21] show that metoprolol can reduce heart rate without detrimental effects on cardiovascular function. During the 96-hour observation period, $97.5 \%$ of patients treated with metoprolol and milrinone had a decrease in heart rate to the target of 65 to 95 beats per minute, a decrease in the central venous pressure (CVP) and an increase in SV index. Mean arterial blood pressure increased despite decreasing norepinephrine, arginine vasopressin and milrinone dosages. These changes, in association with an unchanged cardiac index and a lower heart rate, translate into an economization of cardiac work and oxygen consumption with possible beneficial effects in terms of lowering the risk of myocardial ischaemia and prevention of septic cardiomyopathy. The importance of these observations deserves to be tested prospectively.

In summary, in the management of septic shock:

1. Normalization of the gastric $\mathrm{pHi}$ within 24 hours of resuscitation is associated with therapeutic success.

2. Phenylephrine does not worsen hepatosplanchnic perfusion during initial haemodynamic support of patients with septic shock.

3. Titration of vasopressors using closed-loop control based on fuzzy logic principles leads to a reduction in total vasopressor dose.

4. Haemodynamic responsiveness, reflected by an increase in SV index, due to dobutamine may predict survival.

5. Metoprolol can reduce heart rate without detrimental effects on cardiovascular function in patients receiving milrinone.

\section{Haemodynamic monitoring Non-invasive haemodynamic monitoring}

Estimation of LV filling pressure currently requires invasive measurement of PAOP via the insertion of a pulmonary artery catheter. Vignon and colleagues [22] prospectively assessed the ability of transoesophageal echocardiography (TEE) to predict an invasive PAOP of not more than $18 \mathrm{~mm} \mathrm{Hg}$ in ventilated patients. TEE Doppler parameters were compared with PAOP in a group of haemodynamically stable ventilated patients in sinus rhythm. The proposed Doppler tissue imaging and colour Doppler indices were then tested prospectively in a second group of patients to determine predictive values for an invasive PAOP of not more than $18 \mathrm{~mm} \mathrm{Hg}$.

Doppler parameters that best predicted an invasive PAOP of not more than $18 \mathrm{~mm} \mathrm{Hg}$ were (a) a mitral early-to-late (E/A) ratio of not more than 1.4 (ratio between the mitral $E$ and $A$ velocity, reflecting the atrial contribution to late diastolic LV filling), (b) pulmonary vein systolic-to-diastolic ratio of greater than 0.65 (of peak systolic-to-diastolic velocities in the pulmonary veins) and (c) a systolic filling fraction of the pulmonary vein of greater than $44 \%$ (ratio of the systolic time-velocity integral and the sum of the systolic and diastolic time-velocity integral of pulmonary vein Doppler). The relationship between Doppler indices and invasive PAOP was closer in patients with LV systolic dysfunction.

Artefact is one of the potential problems of echocardiography, particularly TTE. Karabinis and colleagues [23] conducted an ultrasound study to investigate echocardiographic artefacts in mechanically ventilated patients with lung pathology. In a total of 205 mechanically ventilated patients who had lung atelectasis or pleural effusion or both and who were undergoing transthoracic echocardiography, the authors found an intracardiac artefact, termed 'cardiac-lung mass' effect, in $8.29 \%$. This artefact was due to a mirror image created by lung atelectasis or pleural effusion or both, giving the impression of an intracardiac mass not evident on transesophageal echocardiogram or after the lung pathology had resolved.

Critically ill patients have derangements in circulating blood volume, and accurate assessment of volume status is essential for optimal fluid management. In a prospective cohort study in patients admitted within 72 hours after aneurismal subarachnoid haemorrhage, Hoff and colleagues [24] found that clinical assessment of volume status performed by intensive care nurses using conventional haemodynamic parameters was very poor at predicting circulating blood volume when compared with pulse dye densitometry.

Predicting fluid requirement during sepsis was explored by Celi and colleagues [25]. The investigators applied artificial intelligence using a Bayesian network of physiological variables generated from a high-resolution database of information collected during the first 24 hours in ICU. With the predicted total amount of fluid given during the second 24 hours in ICU used as the outcome, the model accuracy was $77.8 \%$, providing proof to the concept that mining empiric data using artificial intelligence can provide patientspecific and clinical scenario-specific recommendations.

\section{Minimally invasive haemodynamic monitoring}

Commercially available $\mathrm{CO}$ monitors use proprietary algorithms to relate arterial pressure to SV and thus $\mathrm{CO}$ and therefore are variably affected by factors that can affect arterial waveform. In these circumstances, algorithms that calculate the SV based on the characteristics of the arterial waveform may not accurately track changes in $\mathrm{CO}$. One of those clinical circumstances is the presence of raised intraabdominal hypertension (IAH).

Using nine haemodynamically stable, fluid-responsive pigs and bolus transcardiopulmonary thermodilution cardiac 
output $\left(\mathrm{CO}_{\mathrm{TCP}}\right)$ as the reference method, Gruenewald and colleagues [26] studied the ability of continuous cardiac output (CCO) methods based on arterial pressure waveform (pulse contour-derived cardiac output [PCCO] and PulseCO) and pulmonary artery catheter thermodilution $\left(\mathrm{CCO}_{\mathrm{PAC}}\right)$ to detect a change in $\mathrm{CO}$ following a fluid challenge. $\mathrm{CO}$ was measured and compared during four steps of the experimental protocol: (a) at baseline, (b) after a fluid challenge, (c) after induction of IAH by pneumoperitoneum and (d) after a fluid challenge in the presence of $\mathrm{IAH}$.

At baseline, all $\mathrm{CO}$ methods showed acceptable agreement in the increase in $\mathrm{CO}$ following volume loading. However, PulseCO and pre-calibration PCCO $\left(\mathrm{PCCO}_{\text {pre }}\right)$ grossly underestimated $\mathrm{CCO}$ following volume challenge in the presence of $\mathrm{IAH}$ when $\mathrm{CO}$ response to fluid was seen in only $\mathrm{CCO}_{\mathrm{PAC}}$ and $\mathrm{CO}_{\mathrm{TCP}}$. After recalibration, $\mathrm{PCCO}$ was comparable to $\mathrm{CO}_{\mathrm{TCP}}$. There was also a progressive increase in bias $\left(\mathrm{CO}_{\mathrm{TCP}}-\right.$ PulseCO versus $\left.\mathrm{CO}_{\mathrm{TCP}}-\mathrm{PCCO}_{\text {pre }}\right)$ during the experimental protocol in the presence of $\mathrm{IAH}$.

The induction of IAH caused increases in CVP, PAOP and chest wall elastance. Arterial blood pressure increased after fluid challenge only in the absence of IAH. This finding and the mechanical effects of IAH on the arterial elastance could account for the inability of waveform-based $\mathrm{CCO}$ algorithms to accurately track changes in $\mathrm{CO}$ after fluid loading during $\mathrm{IAH}$.

Two recent papers by the same investigators assessed the performance of a later FloTrac/Vigileo ${ }^{{ }^{\mathrm{TM}}}$ system algorithm (software version 1.07; Edwards Lifesciences LLC, Irvine, CA, USA). In both papers, the system was assessed in haemodynamically stable patients with a stable regular heart rate maintained between 80 to 90 beats per minute by fixed external pacing after elective off-pump coronary artery bypass grafting.

In the first paper, Hofer and colleagues [27] compared stroke volume variation (SVV) calculated using the new algorithm $\left(\mathrm{SVV}_{\text {FloTrac }}\right)$ with SVV calculated using the $\mathrm{PiCCO}_{\text {Plus }}{ }^{\mathrm{TM}}$ system $\left(S_{\text {PiCCO }}\right)$ during a blood volume shift manoeuvre, instigated by changing body positioning from a $30^{\circ}$ head-up position to a $30^{\circ}$ head-down position. The manoeuvre resulted in significant increases in SV, global end diastolic volume and CVP and significant decreases in SVV FloTrac $_{1}$ SVV $_{\text {PiCco }}$ and PPV. Among the patients with an increase in SV of greater than 25\% $(58 \%$ of the population), SVV $\mathrm{SVlTT}_{\text {Frac }}$ and $\mathrm{SVV}_{\text {Picco }}$ were $16 \% \pm 4 \%$ and $19 \% \pm 5 \%$, respectively. In patients with an increase in SV of less than $10 \%$, baseline SVV FloTrac and SVV Picco $_{\text {were }}$ $9 \% \pm 2 \%$ and $11 \% \pm 3 \%$, respectively. The optimal thresholds of SVV to predict change in $\mathrm{CO}$ following postural change were an $\mathrm{SVV}_{\mathrm{FloTrac}}$ of $9.6 \%$ and an $\mathrm{SVV}_{\mathrm{PiCCO}}$ of $12.1 \%$, based on analysis of the ROC curve.

In the second paper, Senn and colleagues [28] compared the changes in $\mathrm{CO}$ induced by changes in body positioning using the new algorithm of the FloTrac/Vigileo ${ }^{\mathrm{TM}}$ system with (a) the previous software release (1.03), (b) the PiCCOplus ${ }^{\mathrm{TM}}$ system and (c) the intermittent transpulmonary thermodilution as a reference method. Haemodynamic measurements were performed in a supine position, a $30^{\circ}$ head-up position, a $30^{\circ}$ head-down position and on return to a supine position. Comparative analyses of the various algorithms showed an unacceptably large percentage error in $\mathrm{CO}$ between the old version of the FloTrac/Vigileo ${ }^{\mathrm{TM}}$ system and the thermodilution (percentage error of $37.5 \%$ ). The new modified algorithm for the FloTrac/Vigileo ${ }^{\text {TM }}$ system had a significantly better performance with a reduction of the percentage error and changes in $\mathrm{CO}$ that were comparable to the reference technique. Percentage error must be treated with caution, though, particularly when the reference test precision may differ from that expected. We therefore need to know the percentage error (that is, the precision) of both the new and the reference test.

\section{Experimental studies}

Prostacyclin inhalation is used to treat acute pulmonary hypertension and RV failure. To assess the haemodynamic effects of inhaled iloprost on a pig model of acute hypoxiainduced pulmonary hypertension, Rex and colleagues [29] carried out a prospective randomized placebo-controlled study in which inhalation of iloprost (compared with placebo) resulted in selective pulmonary vasodilation associated with an improvement in global haemodynamics, restoration of LV pre-load and a significant increase in $\mathrm{CO}$.

A reduction in $\mathrm{RV}$ afterload was associated with an apparently paradoxical decrease in RV contractility, which was interpreted as being the result of an indirect mechanism ('homeometric autoregulation') caused by the immediate adaptation of RV contractility to match a drug-induced reduction in RV afterload. Parameters reflecting RV oxygen consumption normalized almost to baseline levels after iloprost treatment. Right coronary artery perfusion pressure (estimated as the difference between systolic arterial pressure and RV systolic pressure) increased, indicating a simultaneous improvement in oxygen supply to the right ventricle. There was no evidence of a direct negative inotropic effect of iloprost.

In summary:

1. Indices derived from TEE can predict an invasive PAOP of not more than $18 \mathrm{~mm} \mathrm{Hg}$ in ventilated patients.

2. Clinical assessment of volume status is very poor at predicting circulating blood volume.

3. Bayesian networks of physiological variables can predict fluid requirement in ICU patients.

4. Waveform-based $\mathrm{CCO}$ algorithms do not accurately track changes in $\mathrm{CO}$ after fluid loading during $\mathrm{IAH}$.

5. Version 1.07 of the FloTrac/Vigileo ${ }^{\mathrm{TM}}$ system algorithm shows better performance in haemodynamically stable patients compared with the previous software. 
6. Iloprost improves global haemodynamics, LV pre-load and $\mathrm{CO}$ without direct negative inotropic effects.

\section{Conclusions}

This review has summarized key research papers published in the fields of cardiology and intensive care during 2008 in Critical Care. The papers reflect a wide range of original studies published in Critical Care and cover aspects of cardiovascular biomarkers in critical illness, haemodynamic management of septic shock and haemodynamic monitoring.

\section{Competing interests}

$\mathrm{LC}$ declares that he has no competing interests. DB acts as a consultant for LiDCO Ltd (Sawston, Cambridge, UK). MT has received research equipment from Hutchinson Technology Inc. (Hutchinson, MN, USA).

\section{References}

1. Nesher N, Alghamdi AA, Singh SK, Sever JY, Christakis GT, Goldman BS, Cohen GN, Moussa F, Fremes SE: Troponin after cardiac surgery: a predictor or a phenomenon? Ann Thorac Surg 2008, 85:1348-1354.

2. Adabag AS, Rector T, Mithani S, Harmala J, Ward HB, Kelly RF, Nguyen JT, McFalls EO, Bloomfield HE: Prognostic significance of elevated cardiac troponin I after heart surgery. Ann Thorac Surg 2007, 83:1744-1750.

3. Lim W, Qushmaq I, Devereaux PJ, Heels-Ansdell D, Lauzier F, Ismaila AS, Crowther MA, Cook DJ: Elevated cardiac troponin measurements in critically ill patients. Arch Intern Med 2006, 166:2446-2454

4. Lim W, Holinski P, Devereaux PJ, Tkaczyk A, McDonald E, Clarke F, Qushmaq I, Terrenato I, Schunemann H, Crowther M, Cook D: Detecting myocardial infarction in critical illness using screening troponin measurements and ECG recordings. Crit Care 2008, 12:R36.

5. Jessup M, Abraham WT, Casey DE, Feldman AM, Francis GS, Ganiats TG, Konstam MA, Mancini DM, Rahko PS, Silver MA, Stevenson LW, Yancy CW: 2009 focused update: ACCF/AHA Guidelines for the Diagnosis and Management of Heart Failure in Adults: a report of the American College of Cardiology Foundation/American Heart Association Task Force on Practice Guidelines: developed in collaboration with the International Society for Heart and Lung Transplantation. Circulation 2009, 119:1977-2016.

6. Coutance G, Le Page O, Lo T, Hamon M: Prognostic value of brain natriuretic peptide in acute pulmonary embolism. Crit Care 2008, 12:R109.

7. Kirchhoff C, Leidel BA, Kirchhoff S, Braunstein V, Bogner V, Kreimeier U, Mutschler W, Biberthaler P: Analysis of $\mathrm{N}$-terminal pro-B-type natriuretic peptide and cardiac index in multiple injured patients: a prospective cohort study. Crit Care 2008, 12:R118.

8. Coquet I, Darmon M, Doise JM, Degres M, Blettery B, Schlemmer B, Gambert P, Quenot JP: Performance of N-terminal-pro-Btype natriuretic peptide in critically ill patients: a prospective observational cohort study. Crit Care 2008, 12:R137.

9. Levitt JE, Vinayak AG, Gehlbach BK, Pohlman A, Van Cleve W, Hall JB, Kress JP: Diagnostic utility of B-type natriuretic peptide in critically ill patients with pulmonary edema: a prospective cohort study. Crit Care 2008, 12:R3.

10. Rivers E, Nguyen B, Havstad S, Ressler J, Muzzin A, Knoblich B, Peterson E, Tomlanovich M: Early goal-directed therapy in the treatment of severe sepsis and septic shock. $N$ Engl J Med 2001, 345:1368-1377.

11. Jansen TC, van Bommel J, Mulder PG, Lima AP, van der Hoven B, Rommes JH, Snellen FT, Bakker J: Prognostic value of blood lactate levels: does the clinical diagnosis at admission matter? J Trauma 2009, 66:377-385.

12. Collaborative Study Group on Perioperative $\mathrm{ScvO}_{2}$ Monitoring: Multicentre study on peri- and postoperative central venous oxygen saturation in high-risk surgical patients. Crit Care 2006, 10:R158.

13. Bracht $H$, Hanggi $M$, Jeker $B$, Wegmuller N, Porta F, Tuller $D$, Takala J, Jakob SM: Incidence of low central venous oxygen saturation during unplanned admissions in a multidisciplinary intensive care unit: an observational study. Crit Care 2007,11: R2.

14. van Beest PA, Hofstra JJ, Schultz MJ, Boerma EC, Spronk PE, Kuiper MA: The incidence of low venous oxygen saturation on admission to the intensive care unit: a multi-center observational study in The Netherlands. Crit Care 2008, 12:R33.

15. Bellomo R, Reade MC, Warrillow SJ: The pursuit of a high central venous oxygen saturation in sepsis: growing concerns. Crit Care 2008, 12:130

16. Puyana JC, Pinsky MR: Searching for non-invasive markers of tissue hypoxia. Crit Care 2007, 11:116.

17. Palizas F, Dubin A, Regueira T, Bruhn A, Knobel E, Lazzeri S, Baredes N, Hernandez G: Gastric tonometry versus cardiac index as resuscitation goals in septic shock: a multicenter, randomized, controlled trial. Crit Care 2009, 13:R44.

18. Morelli A, Ertmer $C$, Rehberg $S$, Lange M, Orecchioni A, Laderchi A, Bachetoni A, D'Alessandro $M$, Van Aken $H$, Pietropaoli $P$, Westphal M: Phenylephrine versus norepinephrine for initial hemodynamic support of patients with septic shock: a randomized, controlled trial. Crit Care 2008, 12:R143.

19. Merouani M, Guignard B, Vincent $F$, Borron SW, Karoubi $P$, Fosse JP, Cohen Y, Clec'h C, Vicaut E, Marbeuf-Gueye C, Lapostolle F, Adnet F: Norepinephrine weaning in septic shock patients by closed loop control based on fuzzy logic. Crit Care 2008, 12: R155.

20. Kumar A, Schupp E, Bunnell E, Ali A, Milcarek B, Parrillo JE: Cardiovascular response to dobutamine stress predicts outcome in severe sepsis and septic shock. Crit Care 2008, 12:R35.

21. Schmittinger CA, Dunser MW, Haller M, Ulmer H, Luckner G, Torgersen C, Jochberger S, Hasibeder WR: Combined milrinone and enteral metoprolol therapy in patients with septic myocardial depression. Crit Care 2008, 12:R99.

22. Vignon $P$, AitHssain A, Francois B, Preux PM, Pichon N, Clavel M, Frat JP, Gastinne H: Echocardiographic assessment of pulmonary artery occlusion pressure in ventilated patients: a transoesophageal study. Crit Care 2008, 12:R18.

23. Karabinis A, Saranteas T, Karakitsos D, Lichtenstein D, Poularas J, Yang C, Stefanadis C: The 'cardiac-lung mass' artifact: an echocardiographic sign of lung atelectasis and/or pleural effusion. Crit Care 2008, 12:R122.

24. Hoff RG, Rinkel GJ, Verweij BH, Algra A, Kalkman CJ: Nurses' prediction of volume status after aneurysmal subarachnoid haemorrhage: a prospective cohort study. Crit Care 2008, 12: R153.

25. Celi LA, Hinske LC, Alterovitz G, Szolovits P: An artificial intelligence tool to predict fluid requirement in the intensive care unit: a proof-of-concept study. Crit Care 2008, 12:R151.

26. Gruenewald M, Renner J, Meybohm P, Hocker J, Scholz J, Bein B: Reliability of continuous cardiac output measurement during intra-abdominal hypertension relies on repeated calibrations: an experimental animal study. Crit Care 2008, 12:R132.

27. Hofer CK, Senn A, Weibel L, Zollinger A: Assessment of stroke volume variation for prediction of fluid responsiveness using the modified FloTrac and PiCCOplus system. Crit Care 2008, 12:R82.

28. Senn A, Button D, Zollinger A, Hofer CK: Assessment of cardiac output changes using a modified FloTrac/Vigileo algorithm in cardiac surgery patients. Crit Care 2009, 13:R32.

29. Rex S, Missant C, Claus P, Buhre W, Wouters PF: Effects of inhaled iloprost on right ventricular contractility, right ventriculo-vascular coupling and ventricular interdependence: a randomized placebo-controlled trial in an experimental model of acute pulmonary hypertension. Crit Care 2008, 12:R113. 